\title{
An Exceptionally Potent Inhibitor of Human CD73
}

\author{
Christine E. Bowman, ${ }^{1} *$ Rafael G. da Silva, ${ }^{2}$ Amber Pham, ${ }^{1}$ Stephen W. Young ${ }^{1}$ \\ ${ }^{1}$ Department of Quantitative Biology, Arcus Biosciences, Inc., 3928 Point Eden Way, Hayward, CA 94545, United States \\ ${ }^{2}$ School of Biology, Biomedical Sciences Research Complex, University of St Andrews, St Andrews, Fife KY16 9ST, United \\ Kingdom
}

Supporting Information Placeholder

\begin{abstract}
We recently reported the initiation of a Phase I clinical trial with AB680, a potent human CD73 inhibitor, being developed for the treatment of solid tumors (NCT03677973). We undertook a detailed kinetic analysis on the interaction between human CD73 and AB680 to determine the mode of inhibition. We found $\mathrm{AB} 680$ to be a reversible, slow-onset competitive inhibitor of human CD73 with a $K_{\mathrm{i}}$ of $5 \mathrm{pM}$. Clinical candidates of this potency are uncommon and deserve special consideration during lead optimization.
\end{abstract}

High concentrations of adenosine in the tumor microenvironment are considered to be immunosuppressive. ${ }^{1}$ A major source of adenosine is the extracellular hydrolysis of adenosine 5'-triphosphate (ATP) to adenosine 5'-monophosphate (AMP) and then adenosine by the successive actions of ecto-nucleoside triphosphate diphosphohydrolase (CD39) and ecto-5'-nucleotidase (CD73). Therefore, inhibition of CD73 to reduce extracellular adenosine has emerged as a compelling therapeutic target in immuno-oncology. ${ }^{1}$

The synthesis of AB680 (Chart 1), an inhibitor of human CD73 (hCD73) that is being developed for the treatment of solid tumors, has recently been reported. ${ }^{2,3} \mathrm{CD} 73$ catalyzes the hydrolysis of AMP to adenosine and inorganic phosphate $\left(\mathrm{P}_{\mathrm{i}}\right)$. AB680 represents the culmination of a traditional medicinal chemistry optimization campaign initiated by pharmacophore mapping based on the crystal structure of hCD73 in complex with alpha, beta-methylene-ADP $(\mathrm{AOPCP})^{4}$ and preliminary structure-activity relationships of N6substituted AOPCP derivatives. ${ }^{5}$ During compound screening and the hit-to-lead process, the half-maximal inhibitory concentration $\left(\mathrm{IC}_{50}\right)$ was the primary metric chosen to guide medicinal chemistry through multiple synthetic iterations. During the final stages of lead optimization that ultimately yielded the clinical candidate (AB680), inhibitor $\mathrm{IC}_{50}$ converged rapidly on the enzyme concentration, suggesting potency could be higher than initially thought.

Chart 1. The chemical structure of AB680.<smiles>CC(Nc1cc(Cl)nc2c1cnn2[C@@H]1O[C@H](COP(=O)(O)CP(=O)(O)O)[C@@H](O)[C@H]1O)c1ccccc1F</smiles>

We confirmed tight-binding of $\mathrm{AB} 680$ by measuring its $\mathrm{IC}_{50}$ at different hCD73 concentrations in the presence of saturating amounts of AMP $(30 \mu \mathrm{M})$ (Figure $1 \mathrm{~A}$ and $\mathrm{B}$, Figure S3). The $\mathrm{IC}_{50}$ values decreased linearly with decreasing hCD73 concentration. $\mathrm{IC}_{50}$ determinations can vary as a function of enzyme concentration as described by eq 1 , where $\mathrm{E}_{\mathrm{t}}$ is total enzyme concentration and $K_{\mathrm{i}}^{\text {app }}$ is the mechanism-dependent apparent enzyme-inhibitor dissociation constant. ${ }^{6}$ Fitting the data with linear regression yielded a $K_{\mathrm{i}}^{\text {app }}$ of $13 \pm 9 \mathrm{pM}$ across three independent experiments (Figure 1B, Figure S3).
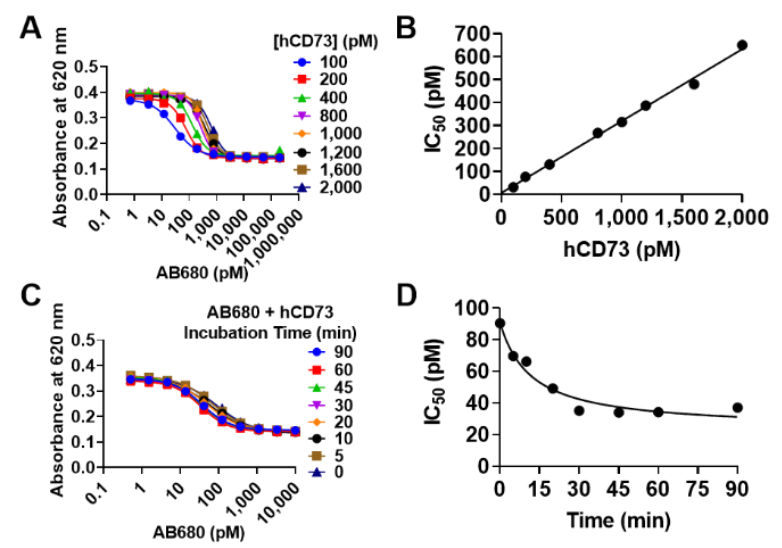

Figure 1. Tight- and slow-binding of AB680 to hCD73. AB680 $\mathrm{IC}_{50}$ dependence on (A and $\left.\mathrm{B}\right)$ concentration of hCD73 and (C and D) incubation time with hCD73. Data in B were fit by linear regression, yielding a slope of 0.31 . Data in $\mathrm{D}$ were fit to eq 3. Panels A and $\mathrm{B}$ are representative of three independent experiments, whereas $\mathrm{C}$ and $\mathrm{D}$, represent two.

Steady-state kinetic parameters for the enzyme under our assay conditions (Figure S2) were $k_{\text {cat }}=46 \pm 3 \mathrm{~s}^{-1}$ and a $K_{\mathrm{M}}=4.1 \pm 0.7$ 
$\mu \mathrm{M}$, resulting in a $k_{\text {cat }} / K_{\mathrm{M}}=(11 \pm 2) \times 10^{6} \mathrm{~s}^{-1} \mathrm{M}^{-1}$. The enzyme concentration was determined from an active site titration (Figure S1) to ensure the active enzyme concentration was as accurate as possible. The enzyme concentration resulting from active site titration with AB680 was repeatedly close to half the concentration initially obtained from protein absorbance at $280 \mathrm{~nm}$. One way to interpret this observation is that approximately half of the active sites are purified in an inactive form. Another, given that hCD73 is a dimer, is that AB680 displays half-of-the-sites inhibition, meaning one mole of inhibitor is enough to inhibit one mole of the dimer. This type of inhibition has been reported, for example, with tightbinding inhibitors of purine nucleoside phosphorylase. ${ }^{7}$

$$
\begin{aligned}
& \mathrm{IC}_{50}=0.5 \times \mathrm{E}_{\mathrm{t}}+K_{\mathrm{i}}^{\mathrm{app}} \\
& \mathrm{P}=(v \times \mathrm{t})-\left(v / k_{\text {obs }}\right) \times\left(1-\mathrm{e}^{-k_{\text {obst }}}\right) \\
& \mathrm{IC}_{50}=\mathrm{IC}_{50}{ }^{\mathrm{min}}+\left(\mathrm{IC}_{50}{ }^{\mathrm{max}}-\mathrm{IC}_{50}{ }^{\min }\right) \times \mathrm{t} /\left(\mathrm{t}+\mathrm{t}_{1 / 2}\right)
\end{aligned}
$$$$
\text { eq } 1
$$

We next evaluated whether AB680 binds reversibly to recombinant hCD73. In a jump-dilution experiment, a 200-fold dilution of an equimolar mixture of hCD73 and AB680 into AMP leads to a slow regain of enzymatic activity, indicating that AMP can eventually outcompete $\mathrm{AB} 680$ for the free enzyme, and that the interaction between enzyme and inhibitor is reversible (Figure 2, Figure S4). There was an initial lag before enzymatic activity was recovered, highlighting the slow dissociation of AB680 from hCD73. Fitting the data to eq 2 , where $\mathrm{P}$ is inorganic phosphate $\left(\mathrm{P}_{\mathrm{i}}\right)$ concentration, $\mathrm{t}$ is time post-dilution, $v$ is the steady-state rate and $k_{\text {obs }}$ is the observed first-order rate constant for dissociation of the EI complex, yielded $k_{\text {obs }}$ of $(2.48 \pm 0.03) \times 10^{-4} \mathrm{~s}^{-1}$.

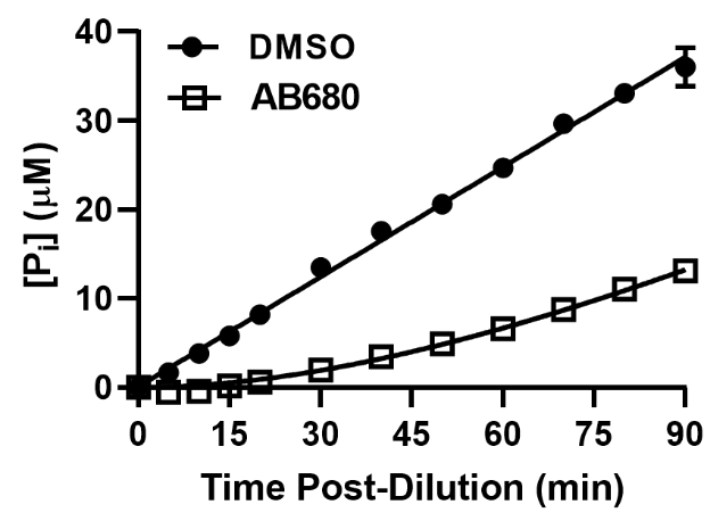

Figure 2. Regain of activity of hCD73. Data were best fit to a straight line (circles) and to eq 2 (squares). The data are representative of two independent experiments.
It is common for inhibitors in this affinity range to display timedependent inhibition. ${ }^{8,9}$ Accordingly, pre-incubation of hCD73 with $\mathrm{AB} 680$ revealed that $\mathrm{IC}_{50}$ s were time-dependent, with increasing pre-incubation times resulting in decreasing $\mathrm{IC}_{50}$ values until a plateau is reached around $30 \mathrm{~min}$ (Figure 1C and D, Figure S5). This suggests that $\mathrm{AB} 680$ does not establish an overall rapid equilibrium with hCD73, but instead binds slowly to the enzyme. The slow-onset inhibition of hCD73 by AB680 is in line with reports for other non-covalent tight-binding inhibitors such as immucillin$\mathrm{H}$, which inhibits purine nucleoside phosphorylase, ${ }^{7}$ and pentacyano(isoniazid)ferrate(II), which inhibits 2-trans-enoyl-ACP reductase. $^{10}$

Three possible kinetic mechanisms have been invoked to explain slow-onset, non-covalent reversible enzyme inhibition (Scheme S1). ${ }^{8}$ The inhibitor may bind to the enzyme in a single, slow step (Scheme S1A), or in two steps, a rapid equilibrium binding followed by a slow conformational change of the enzyme-inhibitor complex (Scheme S1B). A third possibility includes a slow conformational change of the free enzyme, followed by fast inhibitor binding (Scheme S1C).

To distinguish among these three mechanisms, progress curves were determined for the hCD73 reaction at several concentrations of AB680 and analyzed by numerical integration using KinTek Explorer $^{11}$ (Figure 3A). Data fitting in KinTek Explorer was attempted for all three mechanisms, but only the mechanism reflecting Scheme S1A converged on a solution. The following parameters were used to generate the fit: $k_{1}$, describing substrate binding to the enzyme, was constrained within an acceptable range for diffusion-limited binding $\left(100-1000 \mu \mathrm{M}^{-1} \mathrm{~s}^{-1}\right), k_{-1}$, the unimolecular dissociation rate constant for the enzyme-substrate complex, was set to $k_{1} K_{\mathrm{M}}$, which assumes a simple rapid equilibrium between $\mathrm{E}$ and $\mathrm{ES}$, and $k$-2 was set to 0 , since the hydrolytic reaction is irreversible. The remaining parameters were allowed to fluctuate unconstrained during the simulation. This process yielded $k_{3}=73 \pm$ $20 \mu \mathrm{M}^{-1} \mathrm{~s}^{-1}$, the bimolecular rate constant for inhibitor binding to the enzyme, and $k_{-3}=(4 \pm 2) \times 10^{-4} \mathrm{~s}^{-1}$, the unimolecular dissociation rate constant for the enzyme-inhibitor complex, which results in $K_{\mathrm{i}}=5 \pm 3 \mathrm{pM}$. A $k_{2}=49 \pm 1 \mathrm{~s}^{-1}$, the steady-state catalytic constant, was also obtained. Errors reported are the boundaries resulting from FitSpace. The values are remarkably consistent when compared with those obtained from other experiments, with $k_{2}$ in close agreement to $k_{\text {cat }}\left(46 \mathrm{~s}^{-1}\right)$, lending support to the assumption of rapid equilibrium $\left(k_{2}<<k_{-1}\right), k_{-3}$ in close agreement to $k_{\text {obs }}(2.48$ $\times 10^{-4} \mathrm{~s}^{-1}$ ) from the jump-dilution experiment, and $K_{\mathrm{i}}$ in range of $K_{\mathrm{i}}^{\text {app }}(13 \mathrm{pM})$. This lends further support to the proposed inhibition mechanism. Furthermore, multidimensional parameter analysis using FitSpace ${ }^{12}$ showed the values are well-constrained by the model (Figure 3B-D). This is true only for the model comprising a single binding step between inhibitor and enzyme. 

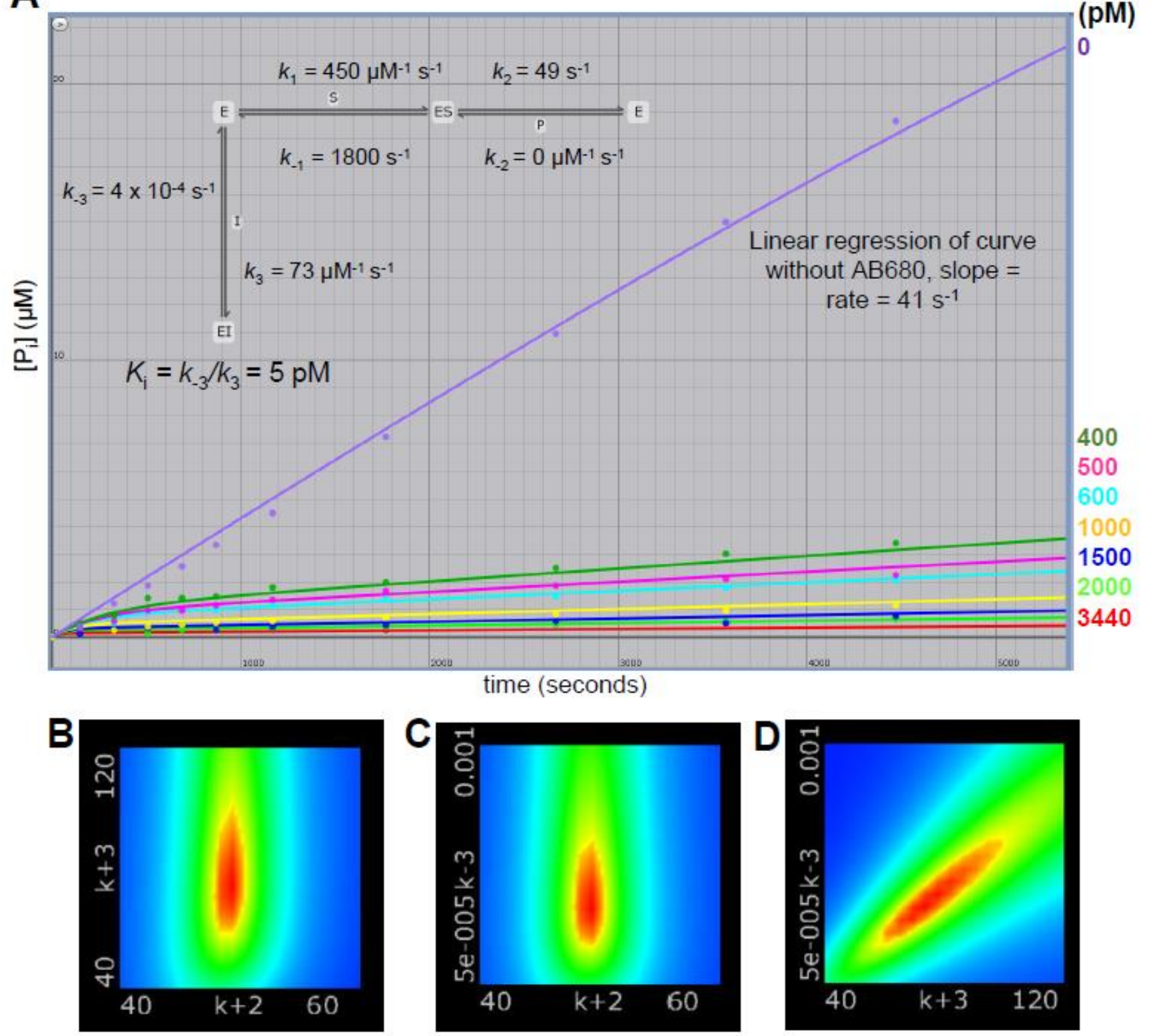

Figure 3. Numerical integration of hCD73 reaction progress curves in the presence and absence of AB680. (A) Data conversion to a singlestep binding mechanism between enzyme and inhibitor. (B-D) FitSpace analysis of the resulting parameters, where the red space delineates the range of values within which the parameters can fluctuate as a function of one another and still be constrained by the model.

$\mathrm{AB} 680$ is an extraordinarily potent, slow-onset, reversible, competitive inhibitor of hCD73. Unlike pentacyano(isoniazid)ferrate(II) which inhibits its target with a two-step binding mechanism ${ }^{13}, \mathrm{AB} 680$ appears to bind hCD73 in a single slow step. This is a similar binding mode to Immucillin- $\mathrm{H}$, which was originally proposed to inhibit its target with a two-step binding mechanism? but upon reanalysis with numerical integration was found to bind in a single slow step. ${ }^{11}$ It should be noted that reports of one-stepbinding slow-onset inhibitors are relatively rare in comparison with those of two-step binding. The inhibition of extracellular signalregulated kinase ${ }^{14}$ and of $\mathrm{p} 38^{15}$ showcase recent examples of onestep-binding slow-onset inhibition. Additionally, specific kinetic factors might lead to the mechanism in Scheme S1B presenting itself as that of Scheme S1A. If the $K_{\mathrm{i}}$ for the first step of the two- step binding mechanism is several orders of magnitude larger $K_{\mathrm{i}}{ }^{*}$ (reflecting the higher affinity for the inhibitor after isomerization of the EI complex), the concentration of the EI complex will be negligible in comparison to that of $\mathrm{EI}^{*}$, and the mechanism will resemble one-step binding in progress curves. ${ }^{9}$ Likewise, the initial bimolecular formation and unimolecular dissociation of the EI complex may be too fast to capture by manual-mixing inhibition progress curve analysis. ${ }^{11}$ Therefore, while numerical integration of the progress curves presented here along with internal consistency with other experiments support one-step binding of AB680 to hCD73, a two-step binding mechanism cannot be entirely ruled out, and future structural and molecular dynamics data, along with pre-steady-state kinetics, may be needed to unequivocally ascertain the mechanism. 
Compounds as potent as AB680 illustrate the need to recognize limitations and adapt quantitative approaches accordingly. This becomes necessary to discover precise kinetic parameters as leads become more potent and inhibitor concentration approaches the enzyme concentration. Since the $K_{\mathrm{i}}$ of AB680 is likely well-below the concentration of hCD73 in vivo, the physiological potency of the drug will be a function of enzyme concentration in the treated tissue, which will have direct implication for dosage.

\section{ASSOCIATED CONTENT}

\section{Supporting Information}

Materials and methods; Figures S1-S5; Scheme S1. The material is available free of charge via the Internet at http://pubs.acs.org.

\section{Accession Code}

hCD73 P21589

\section{AUTHOR INFORMATION}

\section{Corresponding Author}

*Department of Quantitative Biology, Arcus Biosciences, Inc., 3928 Point Eden Way, Hayward, CA 94545. E-mail: cbowman@arcusbio.com

\section{Author Contributions}

C.E.B., R.G.d.S., and S.W.Y. designed the experiments and wrote the manuscript. C.E.B. carried out the experiments. R.G.d.S. fit the progress curves using KinTek Explorer. A.P. purified recombinant hCD73. All authors have given approval to the final version of the manuscript.

\section{Notes}

C.E.B., A.P. and S.W.Y. are employees and stockholders of Arcus Biosciences, Inc.

\section{ACKNOWLEDGMENTS}

We thank all the members of the Arcus team, particularly the Chemistry Department for the synthesis of this molecule, and Ada Chen for initial assay development. We thank Norbert Sträter and Karen Knapp (Leipzig University, Germany) for useful discussions on the expression of hCD73. The research described in this manuscript was fully funded by Arcus Biosciences, Inc. a publicly traded biotechnology company.

\section{ABBREVIATIONS}

AMP, adenosine 5'-monophosphate; ATP, adenosine 5'-triphosphate; $\mathrm{CD}$, cluster of differentiation; $h$, human; $\mathrm{IC}_{50}$, half-maximal inhibitory concentration; $\mathrm{Pi}$, inorganic phosphate; SAR, structure activity relationship; min, minute

\section{REFERENCES}

1. Allard, B., Longhi, M. S., Robson, S. C., Stagg, J. (2017) The ectonucleotidases CD39 and CD73: Novel checkpoint inhibitor targets. Immunol. Rev. 276, 121-144.

2. Lawson, K., Kalisiak, J., Lindsey, E., Newcomb, E., Sharif, E.U., Miles, D., Jeffrey, J., Reddy Leleti, M., Chen, A., Jin, L., Tan, J., Schindler, U., Young, S., Jaen, J., Powers, J. Discovery of AB680, a potent and selective CD73 inhibitor for cancer immunotherapy. 257 ${ }^{\text {th }}$ National Meeting of the American Chemical Society, Orlando, FL. April 3, 2019. Abstract MEDI0257.

3. Debien, L.P.P., Jaen, J.C., Kalisiak, J., Lawson, K.V., Reddy Leleti, M., Lindsey, E.A., Miles, D.H., Newcomb, E., Powers, J.P., Rosen, B.R., Sharif, E.U. U.S. Patent 10,239,912. March 26, 2019.

4. Knapp, K., Zebisch, M., Pippel, J., El-Tayeb, A., Müller, C.E., Sträter, N. (2012) Crystal structure of the human ecto-5'-nucleotidase (CD73): Insights into the regulation of purinergic signaling. Structure. 20, 2161-2173. 5. Bhattarai, S., Freundlieb, M., Pippel, J., Meyer, A., Abdelrahman, A., Fiene, A., Lee, S., Zimmermann, H., Yegutkin, G. G., Sträter, N., ElTayeb, A., Müller, C.E. (2015) $\alpha, \beta$-Methylene-ADP (AOPCP) Derivatives and Analogues: Development of Potent and Selective ecto-5'-Nucleotidase (CD73) Inhibitors. J. Med. Chem. 58, 6248-6263.

6. Copeland, R.A. (2005) Evaluation of enzyme inhibitors in drug discovery. A guide for medicinal chemists and pharmacologists. John Wiley \& Sons, Inc., Hoboken, p.182.

7. Miles, R.W., Tyler, P.C., Furneaux, R.H., Bagdassarian, C.K., Schramm, V.L. (1998) One-third-the-sites transition-state inhibitors for purine nucleoside phosphorylase. Biochemistry. 37, 8615-8621.

8. Morrison, J.F. (1985) Approaches to the study and analysis of the inhibition of enzymes by slow and tight-binding inhibitors. Comments Mol Cell. Biophys. 2, 347-368.

9. Morrison, J.F. and Walsh, C.T. (1988) The behavior and significance of slow-binding enzyme inhibitors. Adv. Enzymol. Relat. Areas Mol. Biol. 61, 201-301.

10. Oliveira, J.S., Sousa, E.H., Basso, L.A., Palaci, M., Dietze, R., Santos, D.S., Moreira, I.S. (2004) An inorganic iron complex that inhibits wild-type and an isoniazid-resistant mutant 2-trans-enoyl- $\mathrm{ACP}(\mathrm{CoA})$ reductase from Mycobacterium tuberculosis. Chem. Comm. 3, 312-313.

11. Johnson, K.A. (2009) Fitting enzyme kinetic data with Kinetic Global Kinetic Explorer. Methods Enzymol. 467, 601-626.

12. Johnson, K.A., Simpson, Z.B., Blom T. (2009) FitSpace Explorer: An algorithm to evaluate multidimensional parameter space in fitting kinetic data. Anal Biochem. 387, 30-41.

13. Oliveira, J.S., de Sousa, E.H., de Souza, O.N., Moreira, I.S., Santos, D.S., Basso, L.A. (2006) Slow-onset inhibition of 2-trans-enoyl-ACP (CoA) reductase from Mycobacterium tuberculosis by an inorganic complex. Curr. Pharm. Des. 12, 2409-2424.

14. Rudolph, J., Xiao, Y., Pardi, A., Ahn, N.G. (2015) Slow inhibition and conformation selective properties of extracellular signal-regulated kinase 1 and 2 inhibitors, Biochemistry. 54, 22-31

15. Millan, D.S., Bunnage, M.E., Burrows, J.L., Butcher, K.J., Dodd, P.G., Evans, T.J., Fairman, D.A., Hughes, S.J., Kilty, I.C., Lemaitre, A., Lewthwaite, R.A., Mahnke, A., Mathias, J.P., Philip, J., Smith, R.T., Stefaniak, M.H., Yeadon, M., and Phillips, C. (2011) Design and synthesis of inhaled $\mathrm{p} 38$ inhibitors for the treatment of chronic obstructive pulmonary disease. J. Med. Chem. 54, 7797-7814. 
Authors are required to submit a graphic entry for the Table of Contents (TOC) that, in conjunction with the manuscript title, should give the reader a representative idea of one of the following: A key structure, reaction, equation, concept, or theorem, etc., that is discussed in the manuscript. Consult the journal's Instructions for Authors for TOC graphic specifications.

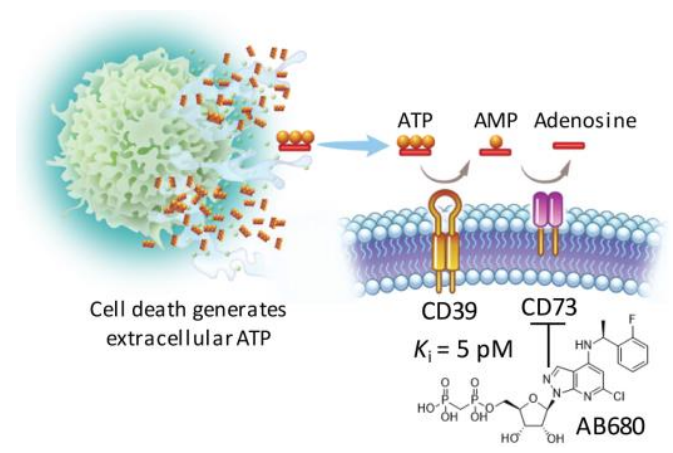

